\title{
Lycopene/Tomato Consumption and the Risk of Prostate Cancer: A Systematic Review and Meta-Analysis of Prospective Studies
}

\author{
Jinyao CHEN, Yang SONG and Lishi ZHANG* \\ West China School of Public Health, Sichuan University, 16", Third Section, \\ South Renmin Road, Chengdu, Sichuan, 610041, P.R. China
}

(Received October 18, 2012)

\begin{abstract}
Summary Lycopene/tomato has been discussed as a potential effecter in the prevention and therapy of prostate cancer; however, no systematic review has been reported to illustrate its effect recently. In the present study, a meta-analysis was carried out to determine whether intake of lycopene and tomato/tomato products could reduce the risk of prostate cancer. Eleven cohort studies and six nested case-control studies were identified through searching of international journal databases and reference lists of relevant publications. Two reviewers independently assessed the study quality and extracted data from each identified study; only studies with sufficient quality were included in the review. The main outcome of interest was incidence of prostate cancer. Compared with consumers of lower raw tomato intake, the odds ratio (OR) of incidence of prostate cancer among consumers of higher raw tomato intake was 0.81 [95\% confidential interval (CI) 0.59-1.10]; for consumers of higher level of cooked tomato intake versus lower cooked tomato intake, this OR was 0.85 (95\% CI 0.69-1.06); the OR of higher lycopene intake versus lower lycopene intake for prostate cancer was 0.93 (95\% CI 0.86-1.01) and the OR for higher level of serum lycopene versus lower serum lycopene level was 0.97 (95\% CI 0.88-1.08). It's suggested that tomato may play a modest role in the prevention of prostate cancer. Further research would be needed to determine the type and quantity of tomato products regarding their potential in preventing prostate cancer.
\end{abstract}

Key Words lycopene, tomato, prostate cancer, meta-analysis, systematic review

Prostate cancer is a common form of cancer with high incidence worldwide, which is also a common cause of death in developed countries with age-standardized mortality rates ranging from 16 per 100,000 males in the U.S. to 28 per 100,000 males in Norway and Sweden (1). As evidenced in the dietary supplements industry, consumers are increasingly turning to diet-based interventions and complementary medicines to maintain health and prevent diseases (2). An investigation in 2005 identified that up to $33 \%$ participants diagnosed with prostate cancer used some form of complementary and alternative products or practice, with an overall rate of $25 \%$ reporting of dietary supplements, such as lycopene (3).

Lycopene, a carotenoid found in high quantities in tomatoes and tomato-rich products, has been explored in association with prevention and treatment of various kinds of diseases, including prevention of prostate cancer. Besides, lycopene has been identified as an antioxidant compound with potential anti-cancer properties and no obvious side effects (4). The American Cancer Society Guidelines on Nutrition and Physical Activity for Cancer Prevention suggested that eating five or more servings of vegetables and fruits (which may include lycopene) each day may protect against prostate cancer;

\footnotetext{
*To whom correspondence should be addressed.
}

E-mail: lishizhang_56@163.com however, the effectiveness is still under investigation (5). In 2004, the FDA received two petitions for qualified health claims regarding tomatoes and/or lycopene and cancer risk reduction, then the scientific data for tomato and/or lycopene intake with respect to risk reduction for certain forms of cancer were reviewed accordingly; however, the FDA found no credible evidence to support an association of lycopene intake with a reduced risk of prostate, lung, colorectal, gastric, breast, ovarian, endometrial, or pancreatic cancer, and very limited evidence to support an association of tomato consumption with reduced risks of prostate, ovarian, gastric, and pancreatic cancers (6). In 2007, the World Cancer Research Fund reported that a high intake of fruit and vegetable may be beneficial in reducing the risk of cancer, including lycopene for prostate cancer (7).

Up to now, it's still controversial to ascertain a role of the nutritional supplementation in the prevention of prostate cancer considering current scientific evidence. In addition, there still remain some unanswered questions, e.g. whether this benefit varies with different preparations of tomatoes (e.g. cooked versus raw), and how closely circulating or tissue lycopene level reflected dietary intake.

To answer some of these questions and provide a more convincing statement, we therefore conducted a systematic review of all available prospective evidence relating to lycopene supplementation/tomato intake 


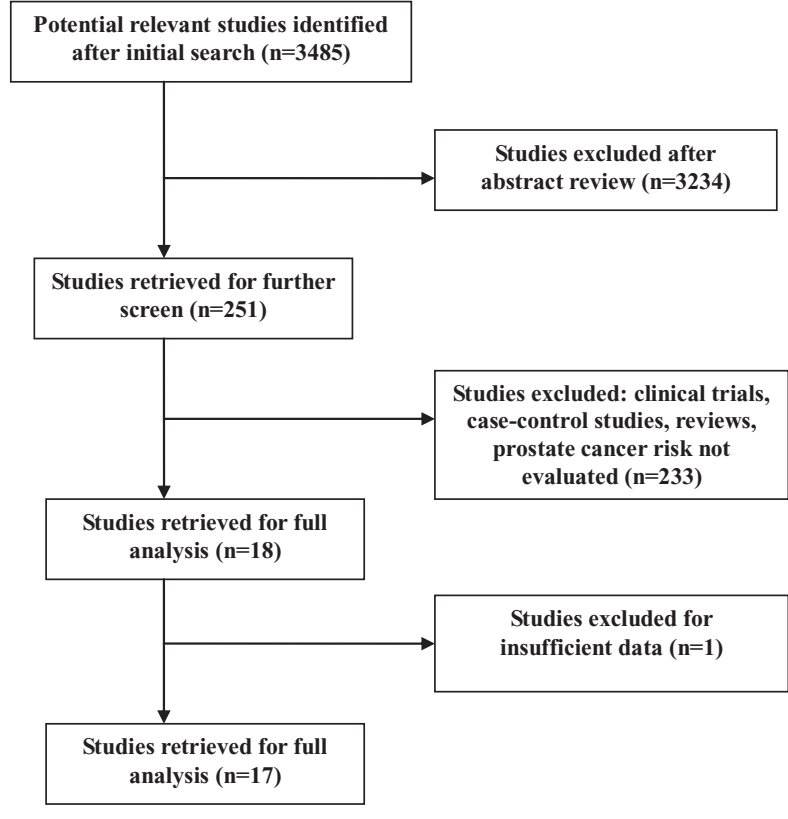

Fig. 1. Search strategy flow chart.

and/or serum lycopene and prostate cancer incidence to provide evidence-based recommendations for populations with a high risk of prostate cancer as well as the general public.

\section{METHODS AND MATERIALS}

Search strategy. OVID MEDLINE, EMBASE, Web of Science, Springer, CNKI and CENTRAL (The Cochrane Library) databases were systematically searched for relevant studies published before January 2012, and the literature reviewed was limited to studies in humans. Bibliographies of published reviews on lycopene and tomato were also screened for potentially relevant studies. A further search was done by scanning the reference lists of original and review publications. No language or other limitations were imposed. Figure 1 illustrates the study selection process.

Terms used for MEDLINE (and adopted for other databases) in both subject heading and text words were: (1) carotenoids or lycopene or antioxidants or tomato or dietary; (2) prostate cancer or prostatic neoplasm; (3) (1) and (2); (4) limit (3) to humans.

Studies were included if they met the following criteria: (1) the primary outcome was clearly defined as prostate cancer; (2) the exposure of interest was tomato consumption or lycopene intake, or measurement of serum lycopene; (3) odds ratio (OR) or relative risk (RR) estimates and their confidence intervals (CIs), or enough data to calculate them was provided; (4) the most confounding factors were adjusted. If data or population were duplicated in more than one study, the most recent study was included in the meta-analysis.

Data extraction and quality assessment. The papers identified through the initial search were reviewed and classified as potentially relevant by two independent reviewers (CJY and SY), data was evaluated and extracted independently from included studies by the two reviewers. Any discrepancy was discussed and resolved in consensus and by consulting with the superior (ZLS).

Data extracted included first author, publication year, study setting, study design, study population, sample size, outcome assessment, duration of observation and follow-up, statistical methods, and risk estimators for the association of different exposure levels of lycopene/ tomato with incidence. The references in relevant publications were checked for additional studies of interest. Missing data was dealt with by contacting the original study investigators to request the missing data, or provide further clarification on data. Analysis was performed on the available data in cases where the missing data was irretrievable.

Quality evaluation was carried out according to the guideline in strengthening the Reporting of Observational Studies in Epidemiology (STROBE) statement to assess the quality of the studies included in this review (8). The quality was independently evaluated for each study by the two reviewers; disagreements were resolved by consensus, and details are described in Tables 1-3.

Statistical analysis. Separate analysis for intakes of raw or cooked tomato, and lycopene with respect to risk of prostate cancer was carried out. Tomato and lycopene intake was reported as categorical data with a range in the studies included in this meta-analysis. An additional analysis focused on serum lycopene in association with prostate cancer.

An overall relative risk for lowest and highest range of lycopene/tomato intake level and serum lycopene was derived by meta-analysis combining estimates from fully adjusted models, and in all instances the ratios used compared the incidence of the highest range of lycopen/ tomato intake and serum lycopene versus the lowest.

A summarized OR with a 95\% CI was estimated by using both fixed-effect and random-effect models. If the data were homogeneous, the fixed-effect model would be applied and if the data were heterogeneous, the random-effect model would be adopted. Given the assumption that lycopene might play a more important role in the prevention of advanced prostate cancer, subgroup analysis was carried out for advanced prostate cancer among studies with available data.

Assessment of heterogeneity was performed using both $\chi^{2}$ and $I^{2}$. The $\chi^{2}$ test assesses whether the differences in results are due to chance only (9). The $I^{2}$ assess the percentage of variability in the effect estimates that is due to heterogeneity rather than chance. $I^{2}$ values over $50 \%$ indicate that substantial heterogeneity may be present (9). Publication bias was assessed via visual inspection of the funnel plots. Sensitivity analysis was performed to strengthen the results of the meta-analysis. The analysis was performed using Review Manager 5.0 software (The Nordic Cochrane Centre, The Cochrane Collaboration, Copenhagen) (10).

\section{RESULTS}

Literature searching and characteristics of included studies The literature search yielded 18 potential prospective 


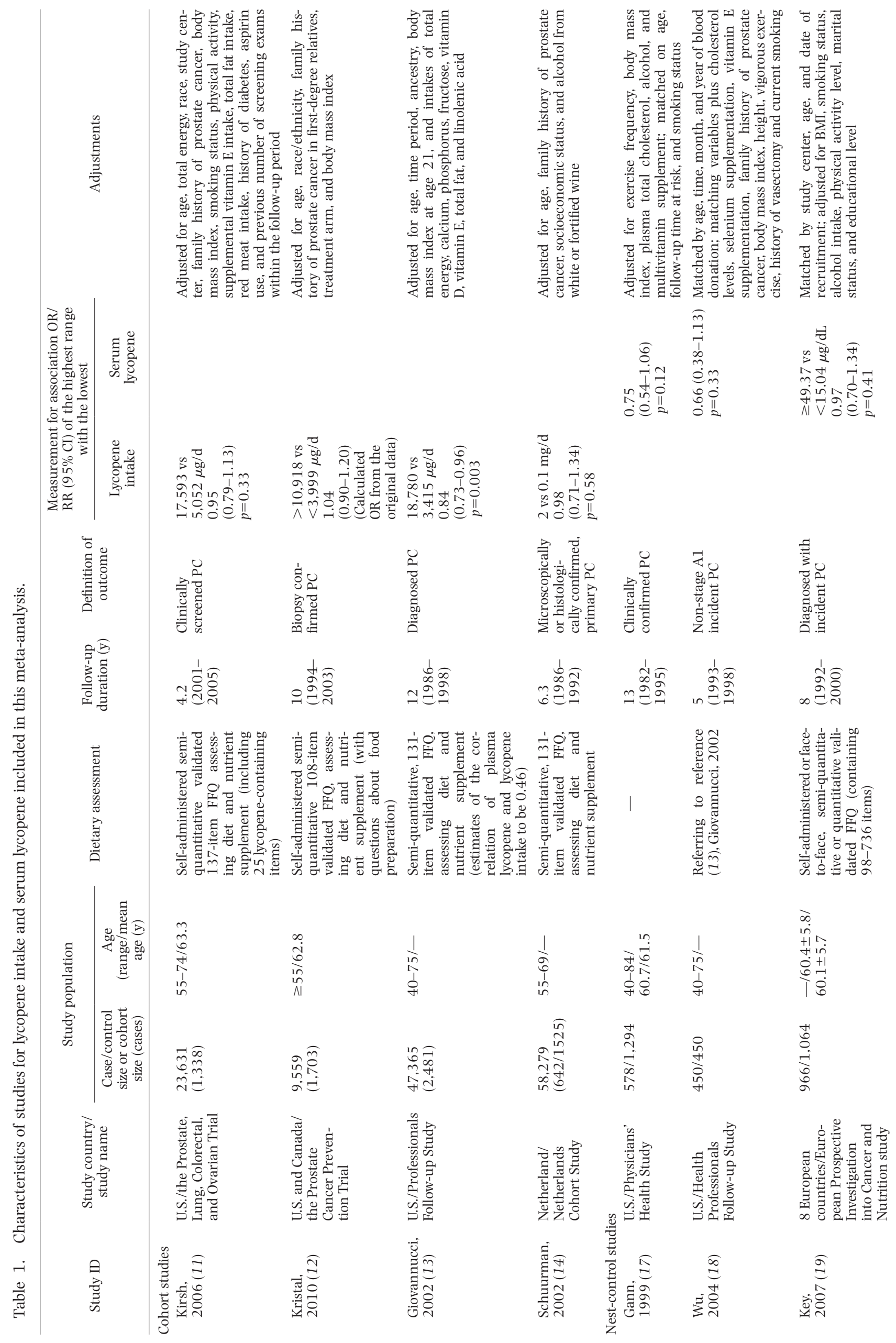




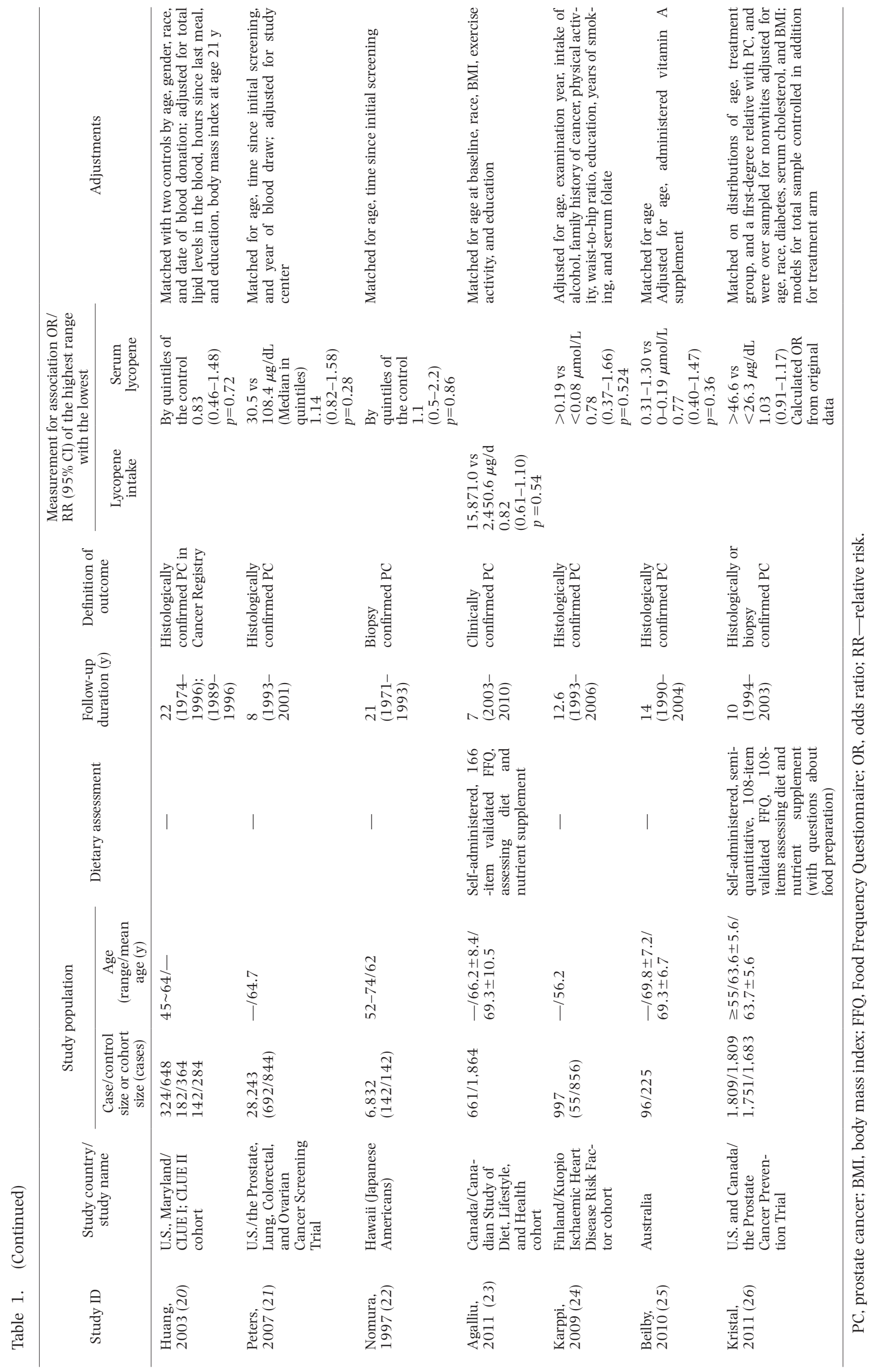


Lycopene/Tomato for Risk of Prostate Cancer

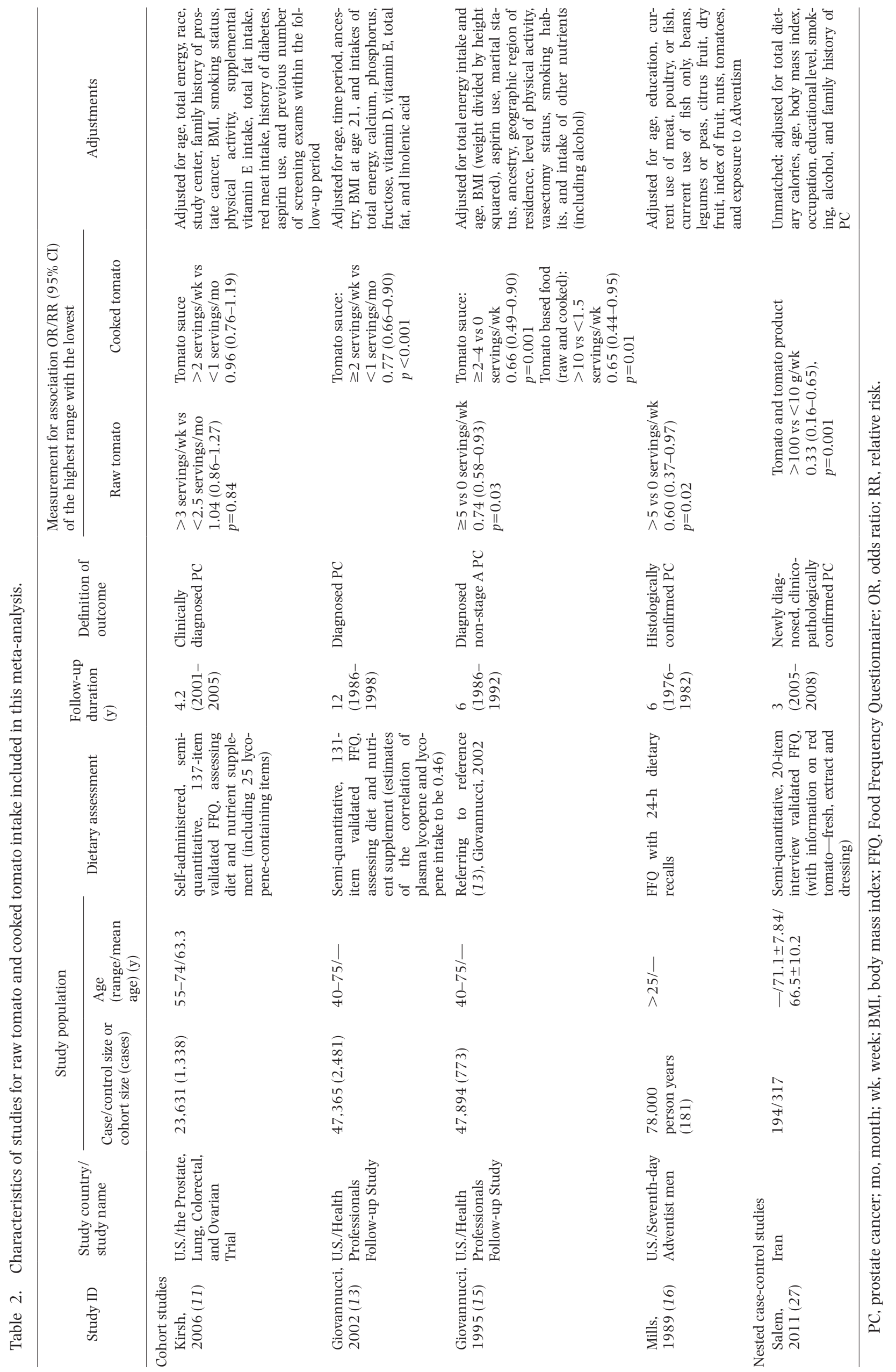




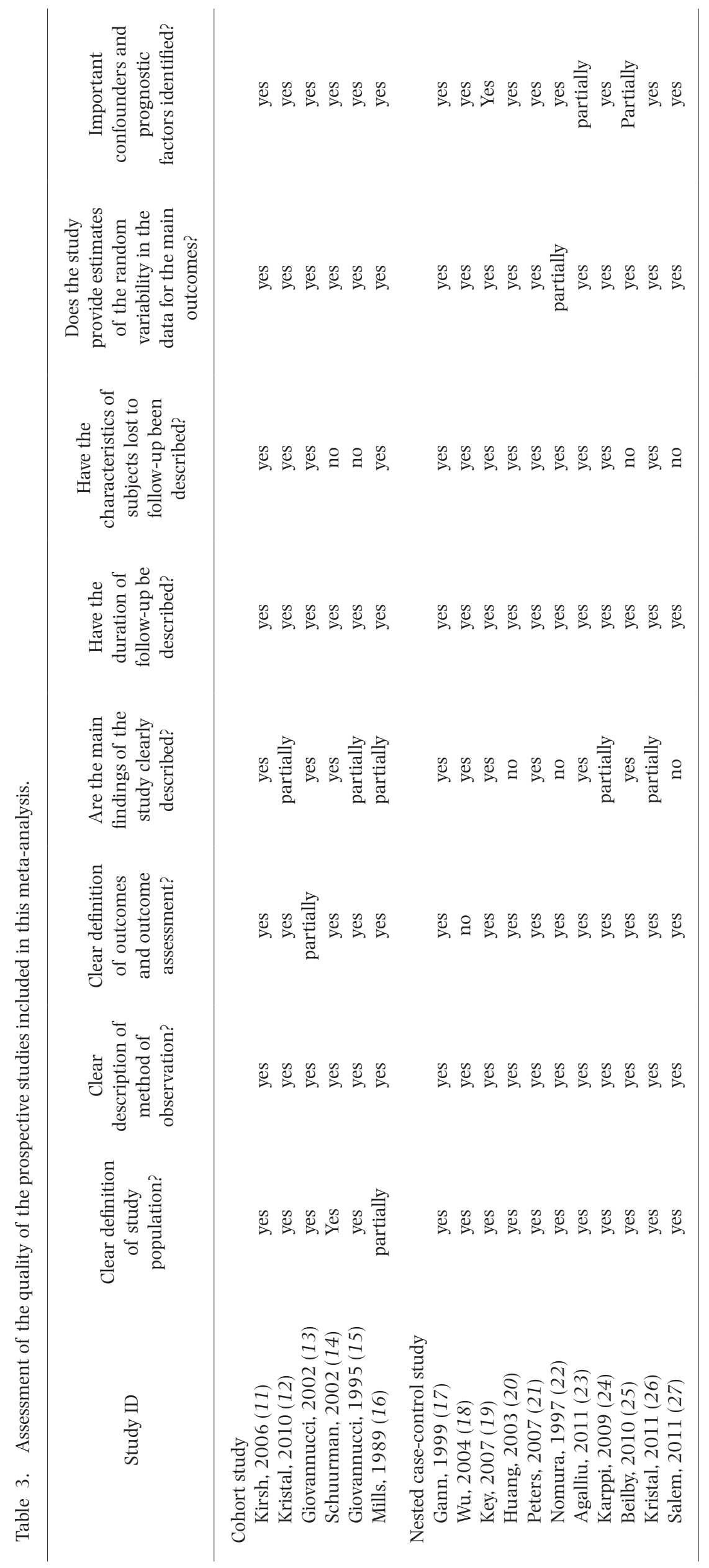




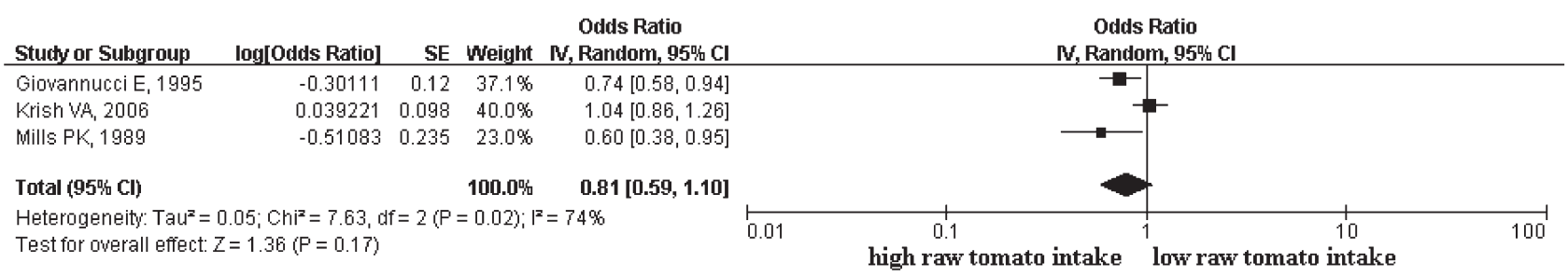

Fig. 2. Effect of raw tomato intake on risk of prostate cancer.

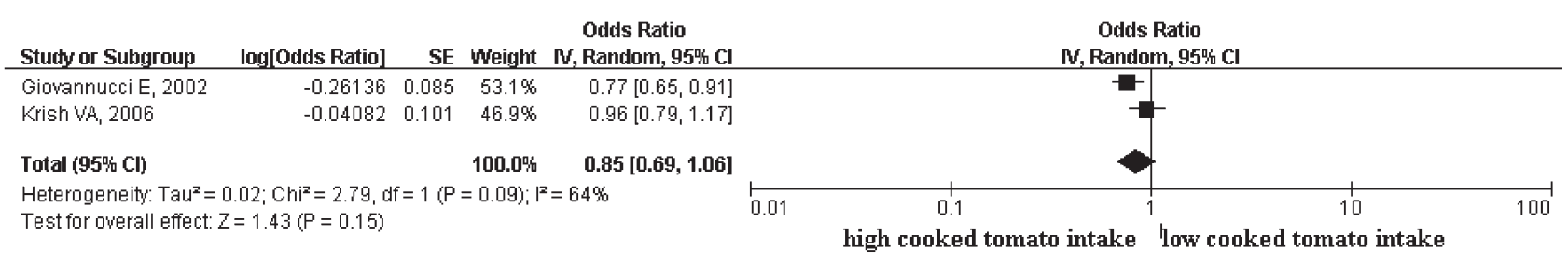

Fig. 3. Effect of cooked tomato intake on risk of prostate cancer.

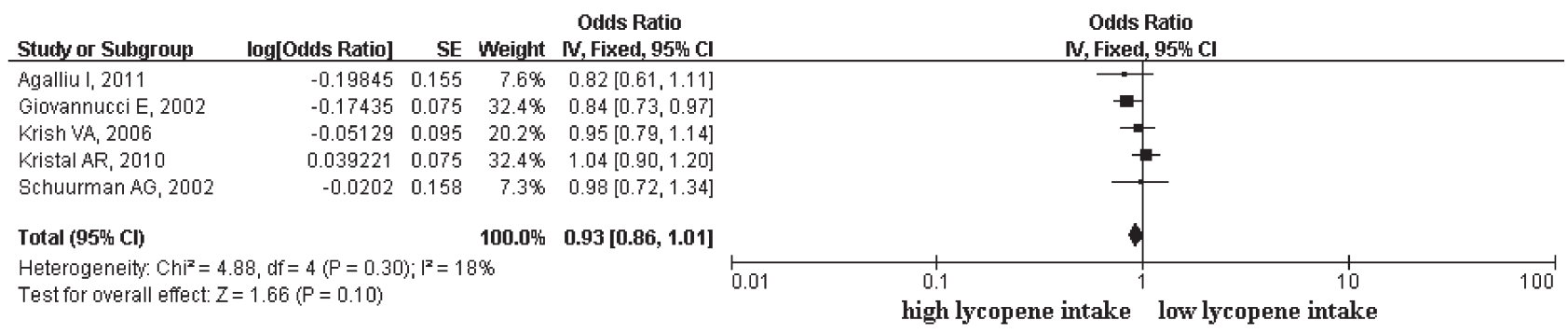

Fig. 4. Effect of lycopene intake on risk of prostate cancer.

studies to be used in the systematic review and finally 17 studies, including six cohort studies (11-16) and 11 nested case-control studies (17-27), were entered in the meta-analysis (Fig. 1). One nested case-control study was excluded as it did not provide CIs of the OR or any other information that allowed for its calculation (28). The authors were contacted by e-mail trying to retrieve those missing data, but up to the time of completion of this article, we had not received this information. Data of one cohort study (29) were included in another follow-up cohort study (20), while the most recent reports were chosen for this review, and results of one cohort (Health Professionals Follow-up Study, HPFC) had been reported in both 1995 and $2002(13,15)$. However, raw tomato intake was only analyzed in 1995 (15); thus updated data about lycopene and cooked tomatoes was adopted from the 2002 analysis (13). The characteristics of the included studies are listed in Tables 1 and 2 .

A food frequency questionnaire (FFQ) was the usual method used to collect dietary intake information. In the cohort studies, dietary intake was captured at the time of enrollment in the study, with the follow-up period ranging from 4.2 to $22 \mathrm{y}$. In HPFC (15), dietary intake was adopted as the cumulative average updated using the average of all the diet questionnaires available to that point in the follow-up period $(1986,1990,1994)$.

Three cohort studies $(11,15,16)$ presented data on raw tomato intake and one nested case-control study
(27) reported unspecified tomato intake. Three cohort studies $(11,13,15)$ presented data for cooked tomatoes. As for lycopene, four cohort studies (11-14) and one nested case-control study (23) presented data on lycopene intake, while nine nested case-control studies (17-22, 24-26) presented results referring to serum lycopene level which was measured at the time of enrollment.

Raw tomatoes and cooked tomatoes with prostate cancer

As shown in Fig. 2, compared with people having the lowest range of consumption of raw tomatoes, the risk estimate of prostate cancer of those having highest range of intake was 0.81 (95\% CI 0.59-1.10), $\chi^{2}=7.63, \mathrm{df}=2, p=0.03, I^{2}=74 \%$.

The pooled risk estimate for all four prospective studies on cooked tomato intake was 0.85 (95\% CI 0.69-1.06), $\chi^{2}=2.79, \mathrm{df}=1, p=0.09, I^{2}=64 \%$ (Fig. 3). Since no universally accepted converted serving for cooked tomato products was available, and the four studies investigated multiple categories of cooked tomato, e.g. pizza, tomato sauce, etc. $(11,15)$ and tomato sauce $(13)$, the risk associated with specific categories of cooked tomatoes could not be calculated. Therefore, the pooled OR of cooked tomatoes was conducted based on the data on tomato sauce, which contributes a considerable amount of cooked tomato consumption.

Lycopene intake and serum lycopene

Compared with people having the lowest range of 


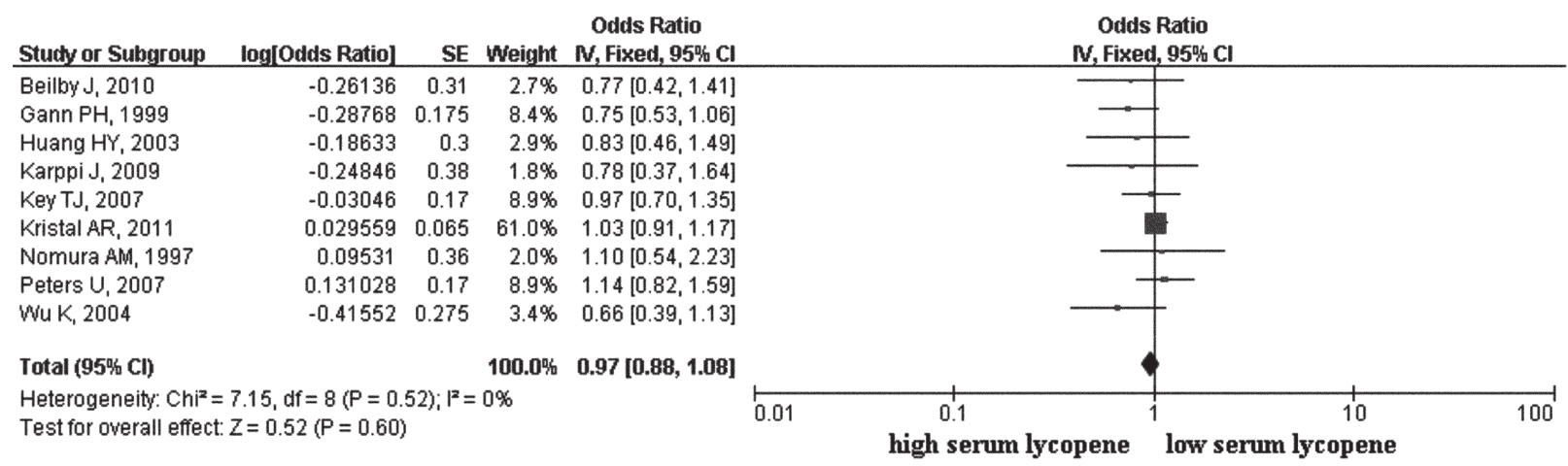

Fig. 5. Effect of serum lycopene on risk of prostate cancer.

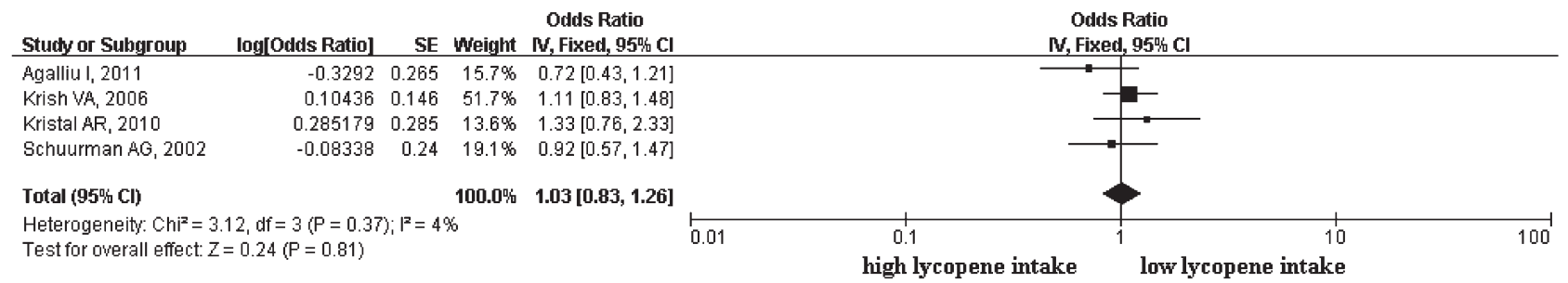

Fig. 6. Effect of lycopene intake on risk of advanced prostate cancer.

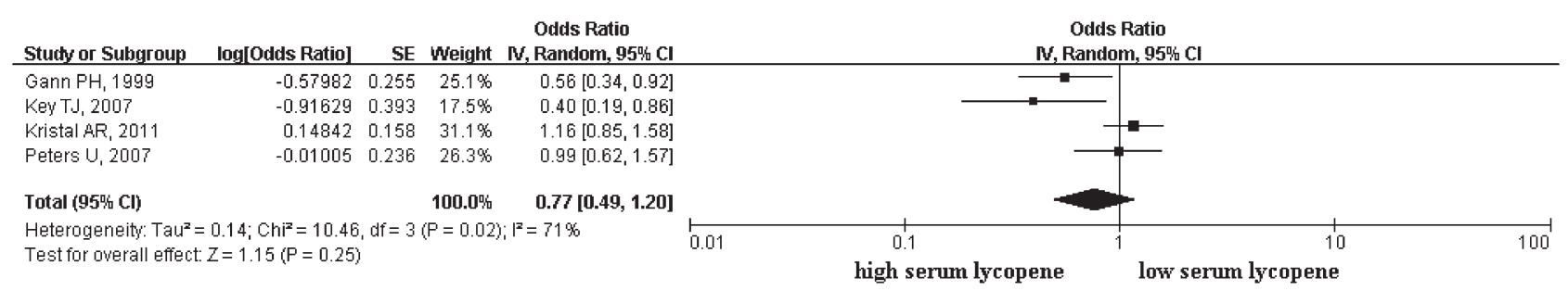

Fig. 7. Effect of serum lycopene on risk of advanced prostate cancer.

consumption of lycopene, the OR of prostate cancer of those having highest intake was 0.93 (95\% CI 0.861.01), $\chi^{2}=4.88, \mathrm{df}=3, p=0.30, I^{2}=18 \%$ (Fig. 4). The effect-estimate shown by increasing lycopene intake was slightly higher than that of concentrations of serum lycopene; as for the highest range of serum lycopene level compared with the lowest, the pooled risk estimate was 0.97 (95\% CI 0.88-1.08), $\chi^{2}=7.15, \mathrm{df}=8, p=0.52$, $I^{2}=0 \%$ (Fig. 5).

Subgroup analysis for advanced prostate cancer

One cohort study-HPFC $(13,15)$-reported the correlation of intake of raw tomatoes and cooked tomatoes with risk of advanced prostate cancer. RR for advanced prostate cancer (stage $C$ and D or Gleason score $\geq 7$ ) of combined intake of tomatoes, and cooked tomato products (which accounted for $82 \%$ of lycopene intake) was 0.65 (95\% CI 0.44-0.95) for consumption frequency greater than 10 versus less than 1.5 servings per week.

Three cohort studies $(11,12,14)$ and one nested casecontrol study (23) reported the risk estimate of lycopene intake with advanced prostate cancer. As shown in Fig. 6 , the pooled estimate for the four studies on lycopene intake with risk of advanced prostate cancer was 1.03
(95\% CI 0.83-1.26), $\chi^{2}=3.12, \mathrm{df}=3, p=0.37, I^{2}=4 \%$. Excluding the nested case-control study (23), the pooled risk estimate of the three cohort studies was $1.10(95 \%$ CI 0.88-1.37), $\chi^{2}=1.00, \mathrm{df}=2, p=0.61, I^{2}=0 \%$.

Four nested case-control studies have reported correlation of serum lycopene with advanced prostate cancer $(17,19,21,26)$. The OR of the highest serum lycopene with the lowest in association with risk of advanced prostate cancer was 0.77 (95\% CI 0.49-1.20), $\chi^{2}=10.46$, $\mathrm{df}=3, p=0.02, I^{2}=71 \%$ (Fig. 7 ).

Publication biases

Funnel plots (Fig. 8) for lycopene intake of five studies and for serum lycopene of nine studies for risk of prostate cancer were consistent with a symmetrical funnel with a large base and a sharp upper part, showing that there is no evidence for publication bias. However, funnel plots were not graphed due to the small number of included studies referring to raw tomatoes and cooked tomatoes and subgroup studies.

Sensitivity analysis

In an attempt to identify the studies contributing to the heterogeneity, a stepwise exclusion of each of the study results for lycopene intake on overall pros- 


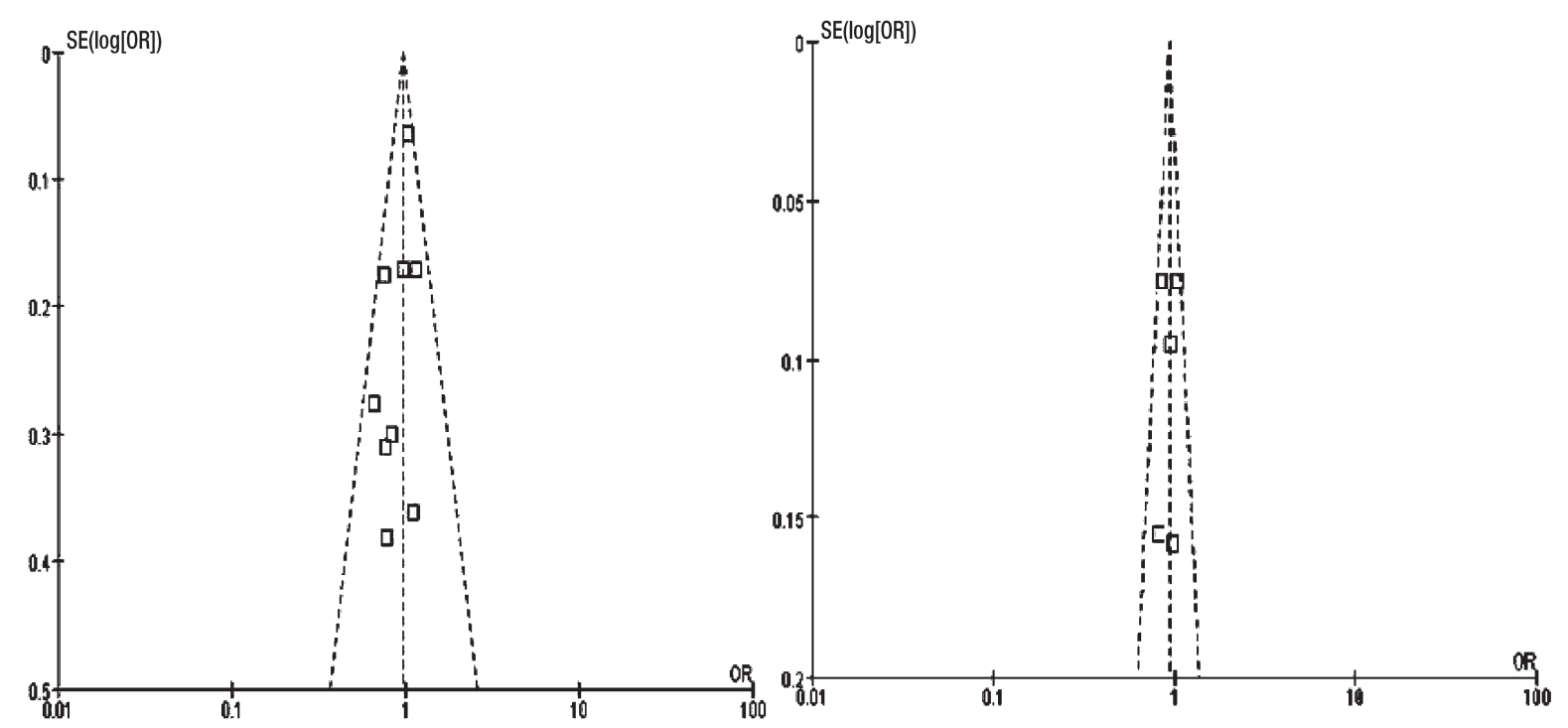

Fig. 8. Funnel plot for serum lycopene for risk of prostate cancer (left) and lycopene intake for risk of prostate cancer (right).

tate cancer was conducted. The risk estimate for lycopene intake was 0.88 (95\% CI $0.80-0.98), \chi^{2}=1.70$, $\mathrm{df}=3, p=0.64, I^{2}=0 \%$ and $0.98(95 \%$ CI $0.88-1.08)$, $\chi^{2}=2.06, \mathrm{df}=3, p=0.56, I^{2}=0 \%$ for excluded study (12) and (13) respectively.

No sensitivity analysis was conducted on tomato intake or subgroup analysis due to limited number of included studies.

\section{DISCUSSION}

Summary of main results

Six cohort studies and 11 nested case-control studies reported from 1989 to 2011 were included in this metaanalysis. To sum up, the inverse associations of intake of raw tomatoes/cooked tomatoes, lycopene intake/serum lycopene with prostate cancer, as well as advanced prostate cancers, were all suggestive but statistically non-significant. Though it has been suggested that the higher concentration and bioavailability of lycopene in cooked tomato products might lead to a more beneficial health outcome, our finding didn't quite support the assumption.

This is the first systematic review and meta-analysis of prospective studies that focused on the effect of lycopene/tomato intake and serum lycopene in association with prostate cancer. The most relevant databases were searched, and a hand search of relevant reviews was also included, with two reviewers independently appraising the evidence quality and extracting data from included studies. Moreover, no publication year or other limitations were restricted. This study incorporated information from studies examining lycopene and tomato intake as well as serum lycopene to illustrate their effect on prostate cancer more clearly.

The quality of the evidence was assessed using the approach outlined in Tables 1-3. It's noteworthy that all the included prospective studies have adjusted data for possible confounding factors, and provided estimates of random variability in the data for the main outcomes and useful information to evaluate the reliability of the studies, such as follow-up approaches, missing data, and dietary questionnaire descriptions. What also should be noted is that FFQ is the most widely adopted method to evaluation lycopene intake. Dietary assessment of lycopene intake may not reflect bioavailability as its absorption may be influenced by several factors including processing or cooking, the lipid content of the diet, and possibly genetic factors $(30,31)$. To date, there is no existing consensus for measuring lycopene intake or for developing a qualified FFQ, which complicated the interpretation of findings across studies. There was large variability in the number of items that were included in the FFQs: some studies captured as few as 20 items (27), while others incorporated up to 736 (19). It's quite possible that questionnaires do not always capture true variation in the lycopene status in a given population, which could result in misclassification and contribute to the notable heterogeneity. Moreover, as the duration of cohort studies generally stretches over years or even decades, the FFQ may ultimately become somewhat 'out-of-date' as new foods become available over the duration of the study and dietary patterns change (32). Therefore, consideration must be given to the possibility of attenuated risk estimates due to random error in quantifying and/or capturing the most relevant measure of intake.

This meta-analysis may further be subject to several limitations. Although there was little evidence of publication bias from the funnel plot, results from the studies that were not accepted for publication apparently may have changed the results of this meta-analysis. Heterogeneity was found among the studies of high intake of raw tomatoes $\left(p=0.03, I^{2}=74 \%\right)$, cooked tomatoes $\left(p=0.09, I^{2}=64 \%\right)$ on prostate cancer, and serum lycopene on risk of advanced prostate cancer $(p=0.02$, $\left.I^{2}=71 \%\right)$. The heterogeneity could be attributed to sev- 
eral factors. Recall bias may be present in dietary studies of tomato/lycopene intake included in our meta-analysis. In an attempt to be comprehensive and include as many qualified studies as possible, we included studies published in countries with various diet pattern and socio-cultural habits [European countries (14, 19, 24, 25), U.S. and Canada (11-13, 15-18, 20-23, 26), and Iran (27) and one specifically targeted in JapaneseAmericans in Hawaii (22)]. Different countries consume different sources of lycopene and different food choice combinations, which may broaden the generalizability of the results, as well as contribute to the heterogeneity of some of the results. Few of the studies made adjustments for a large percentage of the factors (alcohol for instance) found to be important to the pathogenesis of prostate cancer. This may contribute to the heterogeneity in some of the analyses. Duration of follow-up years may also contribute to the heterogeneity, especially what was noted in serum lycopene analysis, given that the included blood-based studies relied on one plasma measurement, which may lead to misclassification of long-term exposure.

Residual confounding (confounding from unknown variables that is not eliminated by adjustment), as in any meta-analysis of observational studies, may introduce considerable bias, and the direction of this bias was probably unpredictable. Moreover, defining what represented a higher intake of lycopene/tomatoes is difficult as there is variability in the upper grade of lycopene/ tomato intake used in the studies, as well as serum lycopene level.

Implication for practice and research

This systematic review has been undertaken to evaluate available prospective evidence for a protective effect of lycopene/tomatoes intake on occurrence of prostate cancer in order to provide evidence-based recommendations. In a meta-analysis of 5 cohorts studies, 5 nested case control studies and 11 case control studies published from the 1950s to 2003 (33), Etminan et al. concluded that there was a $1 \%$ relative risk reduction in the risk of prostate cancer diagnosis in men consuming a higher level of lycopene. To date, more updated and significant results of cohort studies have been reported; therefore, this systematic review would communicate more valuable information.

Though not strongly supporting the positive association of lycopene/tomato intake with reducing risk of prostate cancer, the above meta-analysis does, however, provide reasonable evidence that research on lycopene/ tomato intake in association of prostate health should be carried out among more geographical and age groups and correlation of lycopene intake and plasma lycopene should be examined. Given that existing evidence is not overwhelming enough to recommend the use of lycopene supplements in the prevention of prostate cancer, and referring to the joint statement by World Cancer Research Fund/American Institute for Cancer Research (7), consumption of vegetables and fruits should still be recommended both for cancer prevention and for cancer survivors.

\section{Acknowledgments}

The authors declare that there was no conflict of interest. This work was supported by the National Natural Science Foundation of China (Grant Number 81030053) and the National High-technology Research and Development Program ("863" program) of China (Grant Number 2010AA023001).

\section{REFERENCES}

1) AIHW (Australian Institute of Health and Welfare) \& AACR (Australasian Association of Cancer Registries). 2007. Cancer in Australia: an overview. 2006. Cancer series no. 37. cat. no. CAN 32. AIHW, Canberra.

2) Beebe-Dimmer JL, Wood DP, Gruber SB, Douglas JA, Bonner JD, Mohai C. 2004. Use of complementary and alternative medicine in men with family history of prostate cancer: a pilot study. Urology 63: 282-287.

3) Chan JM, Elkin EP, Silva SJ, Broering JM, Latini DM, Carroll PR. 2005. Total and specific complementary and alternative medicine use in a large cohort of men with prostate cancer. Urology 66: 1223-1228.

4) Rackley JD, Clark PE, Hall MC. 2006. Complementary and alternative medicine for advanced prostate cancer. Urol Clin North Am 33: 237-246.

5) Byers T, Nestle M, McTiernan A, Doyle C, Currie-Williams A, Gansler T, Thun M. 2002. American Cancer Society. 2001. Nutrition and Physical Activity Guidelines Advisory Committee. American Cancer Society Guidelines on Nutrition and Physical Activity for Cancer Prevention. CA Cancer J Clin 52: 92-119.

6) World Cancer Research Fund/American Institute for Cancer Research. 2007. Food, Nutrition, Physical Activity and the Prevention of Cancer: A Global Perspective. Washington, DC.

7) Kavanaugh CJ, Trumbo PR, Ellwood KC. 2007. The U.S. Food and Drug Administration's evidence-based review for qualified health claims: tomatoes, lycopene, and cancer. J Natl Cancer Inst 99: 1074-1085.

8) von Elm E, Altman DG, Egger M, Pocock SJ, Gøtzschee PC, Vandenbroucke JP. 2008. The strengthening the reporting of observational studies in epidemiology (STROBE) statement: guidelines for reporting observational studies. J Clin Epidemiol 61: 344-349.

9) Higgins JPT, Green S. 2008. Cochrane Collaboration. In: Cochrane Handbook for Systematic Reviews of Interventions. Wiley-Blackwell, Hoboken, NJ.

10) The Cochrane Collaboration. 2008. Review Manager (RevMan). The Nordic Cochrane Centre, Copenhagen.

11) Kirsh VA, Mayne ST, Peters U, Chatterjee N, Leitzmann MF, Dixon LB, Urban DA, Crawford ED, Hayes RB. 2006. A prospective study of lycopene and tomato product intake and risk of prostate cancer. Cancer Epidemiol Biomarkers Prev 15: 92-98.

12) Kristal AR, Arnold KB, Neuhouser ML, Goodman P, Platz EA, Albanes D, Thompson IM. 2010. Diet, supplement use, and prostate cancer risk: results from the Prostate Cancer Prevention Trial. Am J Epidemiol 172: 566-577.

13) Giovannucci E, Rimm EB, Liu Y, Stampfer MJ, Willett WC. 2002. A prospective study of tomato products, lycopene, and prostate cancer risk. J Natl Cancer Inst 94: 391-398.

14) Schuurman AG, Goldbohm RA, Brants HA, Van den Brandt PA. 2002. A prospective cohort study on intake of retinal, vitamins $\mathrm{C}$ and $\mathrm{E}$, and carotenoids and prostate cancer risk. Cancer Causes Control 13: 573-578. 
15) Giovannucci E, Ascherio A, Rimm EB, Stampfer MJ, Colditz GA, Willett WC. 1995. Intake of carotenoids and retinol in relation to risk of prostate cancer. J Natl Cancer Inst 85: 1767-1776.

16) Mills PK, Beeson WL, Phillips RL, Fraser GE. 1989. Cohort study of diet, lifestyle, and prostate cancer in adventist men. Cancer 64: 598-604.

17) Gann PH, Ma J, Giovannucci E, Willett W, Sacks FM, Hennekens CH, Stampfer MJ. 1999. Lower prostate cancer risk in men with elevated plasma lycopene levels: results of a prospective analysis. Cancer Res 59: 1225-1230.

18) Wu K, Erdman JW Jr, Schwartz SJ, Platz EA, Leitzmann M, Clinton SK, DeGroff V, Willett WC, Giovannucci E. 2004. Plasma and dietary carotenoids, and the risk of prostate cancer: a nested case-control study. Cancer Epidemiol Biomarkers Prev 13: 260-269.

19) Key TJ, Appleby PN, Allen NE, Travis RC, Roddam AW, Jenab M, Egevad L, Tjønneland A, Johnsen NF, Overvad K, Linseisen J, Rohrmann S, Boeing H, Pischon T, Psaltopoulou T, Trichopoulou A, Trichopoulos D, Palli D, Vineis P, Tumino R, Berrino F, Kiemeney L, Bueno-de-Mesquita HB, Quirós JR, González CA, Martinez C, Larrañaga N, Chirlaque MD, Ardanaz E, Stattin P, Hallmans G, Khaw KT, Bingham S, Slimani N, Ferrari P, Rinaldi S, Riboli E. 2007. Plasma carotenoids, retinol, and tocopherols and the risk of prostate cancer in the European Prospective Investigation into Cancer and Nutrition study. Am J Clin Nutr 86: 672-681.

20) Huang HY, Alberg AJ, Norkus EP, Hoffman SC, Comstock GW, Helzlsouer KJ. 2003. Prospective study of antioxidant micronutrients in the blood and the risk of developing prostate cancer. Am J Epidemiol 157: 335-344.

21) Peters U, Leitzmann MF, Chatterjee N, Wang Y, Albanes D, Gelmann EP, Friesen MD, Riboli E, Hayes RB. 2007. Serum lycopene, other carotenoids, and prostate cancer risk: a nested case-control study in the Prostate, Lung, Colorectal, and Ovarian Cancer Screening Trial. Cancer Epidemiol Biomarkers Prev 16: 962-968.

22) Nomura AM, Stemmermann GN, Lee J, Craft NE. 1997. Serum micronutrients and prostate cancer in Japanese Americans in Hawaii. Cancer Epidemiol Biomarkers Prev 6: 487-491.

23) Agalliu I, Kirsh VA, Kreiger N, Soskolne CL, Rohan TE.
2011. Oxidative balance score and risk of prostate cancer: Results from a case-cohort study. Cancer Epidemiol 35: 353-361.

24) Karppi J, Kurl S, Nurmi T, Rissanen TH, Paukkal E, Nyyssönen K. 2009. Serum lycopene and the risk of cancer: The Kuopio Ischaemic Heart Disease Risk Factor (KIHD) Study. Ann Epidemiol 19: 512-518.

25) Beilby J, Ambrosini GL, Rossi E, de Klerk NH, Musk AW. 2010. Serum levels of folate, lycopene, $\beta$-carotene, retinol and vitamin $\mathrm{E}$ and prostate cancer risk. Eur J Clin Nutr 64: 1235-1238.

26) Kristal AR, Till C, Platz EA, Song X, King IB, Neuhouser ML, Ambrosone CB, Thompson IM. 2011. Serum lycopene concentration and prostate cancer risk: results from the Prostate Cancer Prevention Trial. Cancer Epidemiol Biomarkers Prev 20: 638-646.

27) Salem S, Salahi M, Mohseni M, Ahmadi H, Mehrsai A, Jahani Y, Pourmand G. 2011. Major dietary factors and prostate cancer risk: a prospective multicenter case-control study. Nutr Cancer 63: 21-27.

28) Comstock GW, Helzsouer KJ, Bush TL. 1991. Prediagnostic serum levels of carotenoids and vitamin $\mathrm{E}$ as related to subsequent cancer in Washington County, Maryland. Am J Clin Nutr 53: 260-264.

29) Hsing AW, Comstock GW, Abbey H, Polk BF. 1990. Serologic precursors of cancer. Retinol, carotenoids, and tocopherol and risk of prostate cancer. J Natl Cancer Inst 82: 941-946.

30) Jenab M, Slimani N, Bictash M, Ferrari P, Bingham SA. 2009. Biomarkers in nutritional epidemiology: applications, needs and new horizons. Hum Genet 125: 507-525.

31) Stahl W, Schwarz W, Sundquist AR, Sies H. 1992. Cistrans isomers of lycopene and beta-carotene in human serum and tissues. Arch Biochem Biophys 294: 173-177.

32) Cade J, Thompson R, Burley V, Warm D. 2002. Development, validation and utilization of food-frequency questionnaires—a review. Public Health Nutr 5: 567-587.

33) Etminan M, Takkouche B, Caamano-Isorna F. 2004. The role of tomato products and lycopene in the prevention of prostate cancer: a meta-analysis of observational studies. Cancer Epidemiol Biomarkers Prev 13: 340-345. 